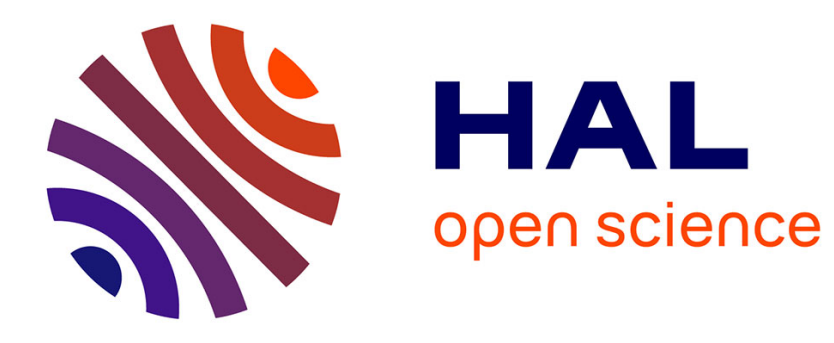

\title{
Étude de résistances de carbone pour la mesure des très basses températures
}

\author{
Albert Lacaze, Romain Peretti
}

\section{To cite this version:}

Albert Lacaze, Romain Peretti. Étude de résistances de carbone pour la mesure des très basses températures. J. Phys. Radium, 1953, 14 (5), pp.350-351. 10.1051/jphysrad:01953001405035001 . jpa-00234749

\section{HAL Id: jpa-00234749 https://hal.science/jpa-00234749}

Submitted on 1 Jan 1953

HAL is a multi-disciplinary open access archive for the deposit and dissemination of scientific research documents, whether they are published or not. The documents may come from teaching and research institutions in France or abroad, or from public or private research centers.
L'archive ouverte pluridisciplinaire HAL, est destinée au dépôt et à la diffusion de documents scientifiques de niveau recherche, publiés ou non, émanant des établissements d'enseignement et de recherche français ou étrangers, des laboratoires publics ou privés. 
déceler une variation de température de l'ordre de $\mathrm{r} / \mathrm{IOOo}^{\mathrm{\theta}}$ de degré $\mathrm{K}$, mais l'état physique du dépôt entraîne une variation isotherme de la résistance, due aux phénomènes d'adsorption des gaz et par conséquent dépendant de la pression résiduelle dans l'enceinte de mesure [2]. D'autre part, Clement et Quinell [3], puis Brown, Zemansky et Boorse [4] ont signalé la possibilité de mesures reproductibles à l'aide de résistances moulées de radio. Les résistancẹs utilisées par ceux-ci étaient choisies parmi un grand nombre de types différents de résistances commerciales. Elles leur permettaient de mesurer la température avec une erreur maximum de o,5 pour ıoo. Cependant, ce procédé se heurte à des difficultés quand on veut mesurer la température d'un échantillon de faible masse ou peu conducteur, eu égard à la capacité calorifique importante du thermomètre. En outre, ces auteurs ont constaté une non-ohmicité notable de ces résistances : par suite du mauvais contact thermique entre le bâtonnet et le corps à étudier, il s'établit en effet un gradient de température dans la résistance quand on fait passer le courant de mesure, gradient qui dépend évidemment de ce courant.

C'est pour pallier à ces difficultés que nous avons été amenés à expérimenter un nouveau type de résistance, étudié et confectionné par l'un de nous (J. P.). La résistance est constituée par une couche mince de graphite pur, déposé à partir d'une dispersion colloïdale dans l'alcool isopropylique (Dag

ETUDE DE RESISTANGES DE CARBONE POUR LA MESURE DES TRÈS BASSES TEMPERATURES

Par Albert Lacaze et Jean Peretri. Laboratoire de Grenoble du C. N. R.S.

Parmi les thermomètres utilisés dans la région de l'hydrogène et de l'hélium liquides, il semble que ceux qui utilisent la variation de résistance du carbone en fonction de la température soient les plus intéressants au point de vue de leur sensibilité et de leur maniabilité.

Giauque et al. [1], utilisèrent en 1938 , pour mesurer la température d'un corps placé dans un champ magnétique, un dépôt de noir de fumée. Le coefficient de température de ce système permet de

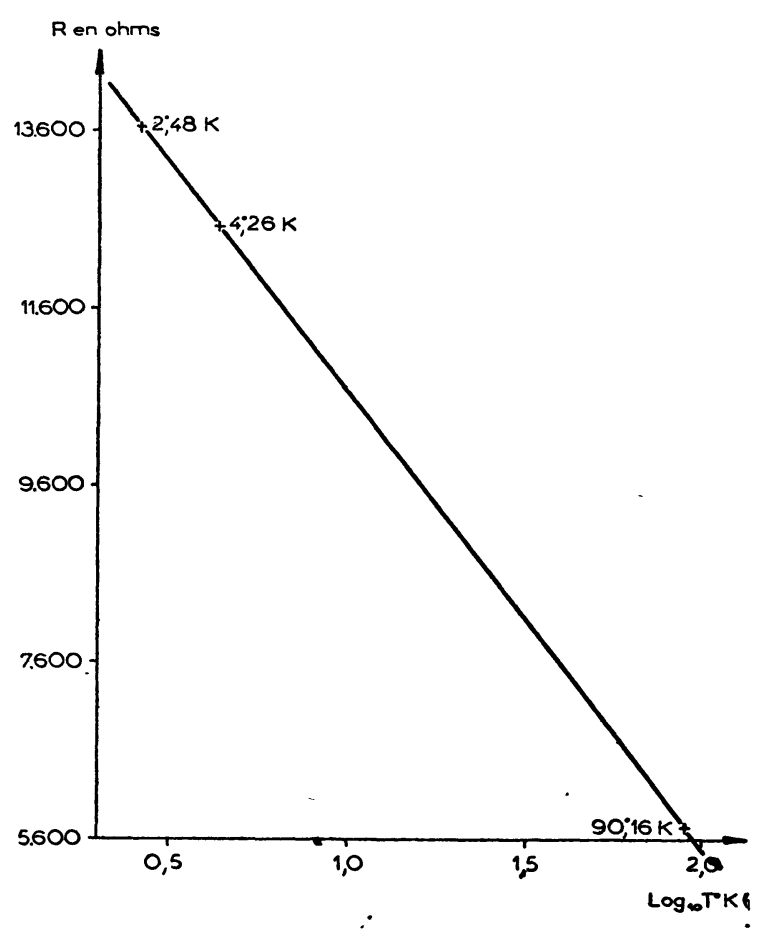

Acheson no 959), sur une céramique dont les extrémités sont argentées chimiquement. Après séchage, cette couche mince est recouverte d'une pellicule de polystyrène. Nous avons essayé également des dépôtśs obtenus à partir de dispersion aqueuse, tels que 
Aquadag, Tilgraph : ils ont un coefficient de température inférieur et il est plus difficile de les recouvrir d'une couche protectrice efficace, par suite d'une mauvaise mouillabilité. Parmi de nombreux revêtements essayés (en particulier l'huile de lin, les esters glycérophtaliques, les esters cellulosiques, la gomme laque et l'araldite) nous avons trouvé que le polystyrène permettait d'obtenir une couche difficilement perméable aux gaz, ce qui limite considérablement les effets d'adsorption et supprime l'effet de pression résiduelle trouvé par Giauque. Cette couche reste plastique même aux très basses températures, comme nous l'a montré l'étude de -la surface au microscope métallographique après retoür à la température ordinaire. D'autre part, la masse du thermomètre est inférieure au $\mathrm{I} / \mathrm{I}_{0} 0_{000^{\circ}}$ de celle du support. Le contact thermique avec l'échantillon de céramique est suffisamment bon pour que l'on puisse certifier l'égalité de température du corps étudié et du thermomètre; notre résistance présente un caractère ohmique normal pour des courants allant jusqu'à $0,2 \mathrm{~mA}$, permettant ainsi d'opérer avec un pont moins sensible que celui de Clement et Quinell.

La sensibilité du dispositif permet d'appré-. cier $0,00 \mathrm{I}^{\circ} \mathrm{K}$ dans l'hydrogène liquide. Pour donner une idée de cette sensibilité nous donnons dans le graphique ci-dessus lesı valeurs mesurées aux températures de l'oxygène liquide sous $76 \mathrm{~cm}$ de $\mathrm{Hg}$, de l'hélium liquide [5] sous $76 \mathrm{~cm}$ et sous $7,4 \mathrm{~cm}$ de $\mathrm{Hg}$, d'une résistance de valeur nominale $3132 \Omega$ à la température ordinaire.
[2] Geball, Lyon, Whelan et Giauque. - Rev. Sc. Instr., $1952,23,23$.

[3] Clement et Quinell. - Ibid., 1952, 23, 2 1 3.

[4] Brown, Zemansky et Boorse. - Phys. Rev., i95r, 84, 1050 .

[5] WeIl L. - J. Physique Rad., r952, 13, 24 1.

[1] Giauque, Stout and Clark. - J. Amer. Chem. Soc., 1938, 60, 1053 .

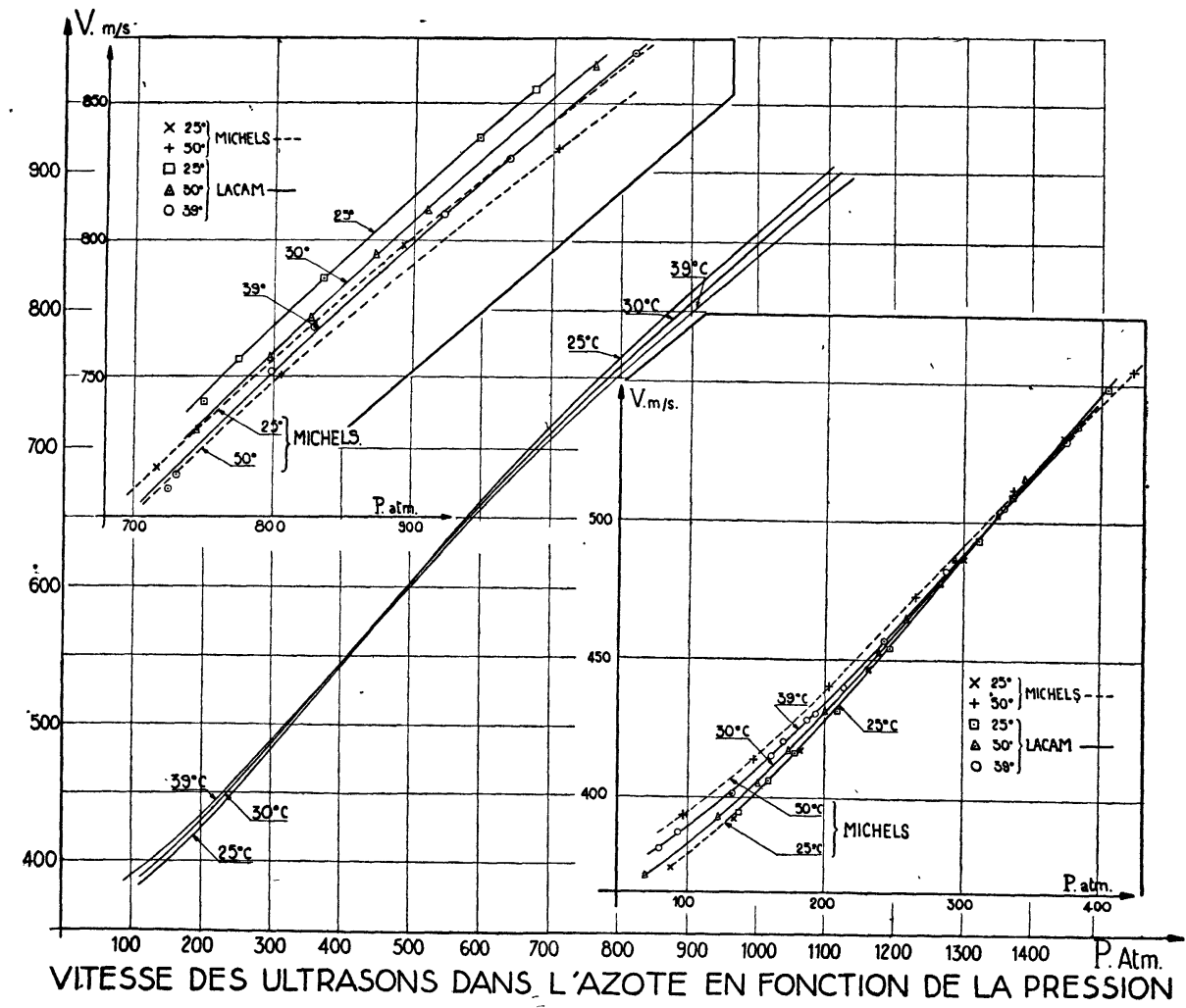

